Available online at GSC Online Press Directory

GSC Biological and Pharmaceutical Sciences

e-ISSN: 2581-3250, CODEN (USA): GBPSC2

Journal homepage: https://www.gsconlinepress.com/journals/gscbps

(REVIEW ARTICLE)

\title{
Characteristics of abdomen and pelvis CT scan's evaluation of patients with malignancies
}

\author{
Natalia Staver* \\ PhD student, Department of Radiology and Medical Imaging "Nicolae Testemitanu” State University of Medicine and \\ Pharmacy from Moldova, 165 Ștefan cel Mare și Sfânt Street, MD-2004, Chișinau, Republic of Moldova.
}

Publication history: Received on 07 August 2020; revised on 08 October 2020; accepted on 12 October 2020

Article DOI: https://doi.org/10.30574/gscbps.2020.13.1.0258

\begin{abstract}
According to the American Cancer Center, cancer causes about 1 in 6 deaths worldwide, more than AIDS, tuberculosis and malaria taken together, it is the second leading cause of death, after cardiovascular disease. Imaging examinations to examine the abdomen and pelvis are the methods of choice in detecting neoplastic formations with the provision of information that is essential for the subsequent management of these patients.

From the PubMed databases and the Google Scholar search engine, the articles published during 2010-2020 were selected, according to the keywords: oncology statistics, oncology imaging, computed tomography, abdominal neoplasms, pelvic neoplasms, oncology staging, post-processing programs in computed tomography, follow-up of cancer patients, diagnostic algorithms. Information on international scientific studies on oncological pathology statistics has been selected and processed globally, according to data from the American Cancer Center and the International Agency for Research on Cancer, innovative methods for assessing the staging of patients with abdominal and pelvic neoplasms, and modern post processing in the case of examination by computed tomography of abdominal and pelvic neoplasms patients.
\end{abstract}

After processing the information in the Google Scholar and PubMed database, according to the search criteria, 346 articles on the proposed topic were found. The final bibliography contains 176 relevant sources, of which 77 were considered representative for the elaboration of this synthesis article.

We must aim to justify, optimize and customize each imaging procedure for patients with neoplasms, as they are frequently exposed to imaging examinations.

Keywords: Oncology statistics; Computed tomography; Abdominal neoplasms; Pelvic neoplasms; Staging in oncology; Post-processing programs.

\section{Introduction}

Cancer is a group of diseases characterized by the uncontrolled growth and spread of abnormal cells. Although the causes of cancer remain largely unknown, many risk factors are known. Some of these are modifiable, such as tobacco use and excess body weight, while others are generally unchangeable, such as inherited genetic mutations, hormones, and immune disorders. These risk factors may act simultaneously or in succession to initiate and / or promote cancer growth.

\footnotetext{
${ }^{*}$ Corresponding author: Natalia Staver

PhD student, Department of Radiology and Medical Imaging "Nicolae Testemitanu" State University of Medicine and Pharmacy from Moldova, 165 Ștefan cel Mare și Sfânt Street, MD-2004, Chișinau, Republic of Moldova.. 
Cancer causes about 1 in 6 deaths worldwide, more than AIDS, tuberculosis and malaria taken together [1]. Today, it is the second leading cause of death, after cardiovascular disease, worldwide (Table 1).

Table 1 Main causes of death worldwide, 2016 (millions)

\begin{tabular}{|c|c|c|c|}
\hline & \multicolumn{3}{|c|}{ Worldwide } \\
\hline & Rank & Death & $\%$ \\
\hline Cardiovascular diseases & 1 & 17,9 & 31 \\
\hline Malignant neoplasms & 2 & 9,0 & 16 \\
\hline Infectious and parasitic diseases & 3 & 5,5 & 10 \\
\hline Respiratory diseases & 4 & 3,8 & 7 \\
\hline Unintentional injuries & 5 & 3,4 & 6 \\
\hline Respiratory infections & 6 & 3,0 & 5 \\
\hline Neurological conditions & 7 & 2,5 & 4 \\
\hline Digestive diseases & 8 & 2,5 & 4 \\
\hline Neonatal conditions & 9 & 2,2 & 4 \\
\hline Diabetes mellitus & 10 & 1,6 & 3 \\
\hline Intentional injuries & 11 & 1,5 & 3 \\
\hline Genitourinary diseases & 12 & 1,4 & 3 \\
\hline Congenital anomalies & 13 & 0,6 & 1 \\
\hline Nutritional deficiencies & 14 & 0,5 & 1 \\
\hline Endocrine, blood, immune disorders & 15 & 0,4 & 1 \\
\hline All cases & & 56,9 & \\
\hline
\end{tabular}

Imaging examinations are the methods of choice in the detection of neoplastic formations that provide essential information for the diagnosis, treatment evaluation and subsequent management of these patients. Currently, the issue of developing standardized imaging techniques, identifying the correct methods for measuring tumor size, data processing and analysis, data collection and image interpretation, in order to make decisions in the strategy of applying appropriate treatment, remains open. Thus in the context of optimizing treatment, identifying and minimizing adverse effects we must adhere to the principle "as low as reasonably achievable" - ALARA, using methods and techniques aimed at optimizing imaging data, minimizing risks and providing the best care clinic of cancer patients.

The researchers contributed to the choice of the effective way of forming protocols and proper image design, they proposed that imaging examinations be performed in stages, each stage having a specific purpose [2]. Imaging monitoring of cancer patients is the basic goal in the evolution of the disease undergoing anticancer treatment for several years after the end of treatment $[3,4,5]$. The Society of Radiologists of North America (RSNA) [6] and the American College of Radiology (ACR) [7] have developed techniques and methods that contain strategic details for assessing the disease's response to therapy and include: ways to determine tumor size measurements, plans in which tumor dimensions measurements are performed, the actual measurement method and the sequences used. All these were designed and executed due to the collaboration and communication between oncologists and radiologistsimagers. Thus, the need for personalization and dynamic imaging evaluation of neoplastic processes response to the 
administered therapy are directly proportional to imaging protocols design and the interpretation of their results. Thus, we can contribute to the study of models aimed at minimizing adverse effects, secondary to imaging diagnosis in the dynamic evaluation of the treatment of cancer patients, which may compromise the quality of life and longevity of these patients.

\section{Material and methods}

In order to achieve the established goal and objectives, a search was performed for the specialized scientific literature, identified by the Google Scholar search engine and from the PubMed database. The articles published during 2010-2020 were selected according to the keywords: statistics in oncology, imaging in oncology, computed tomography (CT), abdominal neoplasms, pelvic neoplasms, staging in oncology, postprocessing programs in computed tomography, follow-up of cancer patients, diagnostic algorithm. Information on international scientific studies on oncological pathology statistics has been selected and processed globally, according to data from the American Cancer Center and the International Agency for Research on Cancer, innovative methods for assessing the staging of patients with abdominal and pelvic neoplasms, and modern postprocessing in the case of examination by CT of patients with abdominal and pelvic neoplasms.

For the advanced search of the desired bibliographic sources, the following filters were applied: articles in English, articles with full text, articles published during the years 2010-2020.

The information in the publications generated by the search engine was classified, synthesized and evaluated. Additional sources of information were consulted to clarify ambiguous notions. Publications and articles that did not correspond to the purpose and objectives of the paper, but also those that could not be accessed for full view, were excluded from the list of publications generated by the search engine.

\section{Results}

According to the International Agency for Research on Cancer (IARC), in 2018, 17.0 million cancer new cases were identified, of which 657,000 in countries with low HDI, 2.8 million in countries with medium HDI , 6.4 million in countries with high HDI and 7.2 million in countries with high level of HDI.

Factors contributing to the incidence of cancer and mortality between countries include variations in age structure, prevalence of risk factors, and availability and use of prevention services, early detection tests, and high-quality treatment (mortality). Many of these factors are strongly influenced by the level of development of the country where these patients live.

The cancer risk increases with age; about $80 \%$ of all cancers in the world are diagnosed in people over the age of 50 [8]. Cancer survival in a population is affected by a number of factors, including the cancer types that occur, the stages in which they are diagnosed, the prevalence of early detection / screening services and whether treatment is available or not.

Substantial evidence supports the causal links between excess body weight and many types of cancer. IARC (International Agency for Research on Cancer) in 2016 concluded that there is sufficient evidence for a causal association between excess body weight and cancer risk of anatomical regions such as: mammary gland (postmenopause), colorectum, uterine body (endometrium), bladder biliary, kidney, liver, multiple myeloma, ovary, pancreas, stomach (cardia) and thyroid gland [9].

According to IARC (International Agency for Research on Cancer), in 2018 in the Republic of Moldova, at a number of $4,041,068$ of the population, 15,284 cancer new cases were registered (in $2013-8,441$ new cases) which represents an rate incidence of 254.3. The number of cases prevalent over the 5 years-period constitutes, in 2018, 32,220 cases. Mortality from malignant tumors in 2018 - 8,508 deaths (in 2013 - 5,835 deaths) representing a mortality rate of 137.1 (Table 2). The risk of developing cancer by the age of 75 is $32.2 \%$ for men and $22.2 \%$ for women, with an average of $25.5 \%$ for both sexes (Table 2). 
Table 2 Summary statistics in the Republic of Moldova, 2018

\begin{tabular}{|c|c|c|c|}
\hline & Males & Females & Both sexes \\
\hline Population & 1.937 .429 & 2.103 .639 & 4.041 .068 \\
\hline Number of new cases & 7.979 & 7.305 & 15.284 \\
\hline Age-standardized incidence rate (world) & 310,5 & 216,6 & 254,3 \\
\hline $\begin{array}{l}\text { Risk of development cancer before the age } \\
\text { of } 75 \text { years }(\%)\end{array}$ & 32,2 & 22,2 & 26,5 \\
\hline $\begin{array}{l}\text { Number of cancer deaths } \\
\text { Age-standardized mortality rate (world) }\end{array}$ & $\begin{array}{l}4.973 \\
191,8\end{array}$ & $\begin{array}{l}3.535 \\
97,7\end{array}$ & $\begin{array}{l}8.508 \\
137,1\end{array}$ \\
\hline $\begin{array}{l}\text { Risk of dying from cancer before the age of } \\
75 \text { years }(\%)\end{array}$ & 21,6 & 11,1 & 15,7 \\
\hline 5-year prelevant cases & 14.589 & 17.631 & 32.220 \\
\hline $\begin{array}{l}\text { Top } 5 \text { most frecquent cancer excluding non- } \\
\text { melanoma skin cancer (ranked by cases) }\end{array}$ & $\begin{array}{l}\text { Lung } \\
\text { Colorectum } \\
\text { Prostate } \\
\text { Liver } \\
\text { Stomach }\end{array}$ & $\begin{array}{l}\text { Breast } \\
\text { Colorectum } \\
\text { Cervix uteri } \\
\text { Corpus uteri } \\
\text { Lung }\end{array}$ & $\begin{array}{l}\text { Colorectum } \\
\text { Lung } \\
\text { Breast } \\
\text { Liver } \\
\text { Prostate }\end{array}$ \\
\hline
\end{tabular}

Source: International Agency for Research on Cancer

In the malignant tumors morbidity-due structure, by location, in 2018, in the Republic of Moldova on the first place is the colorectal cancer with 2,171 new cases, representing 14.2\% (in $2013-12.3 \%$ ), on the second place is lung cancer with 1,707 new cases, representing $11.2 \%$ (in $2013-10.5 \%$ ), on the third place is the cancer of the mammary gland with 1,646 new cases, representing 10.8\% (in 2013 - 11.8\% ) (Figure 2).

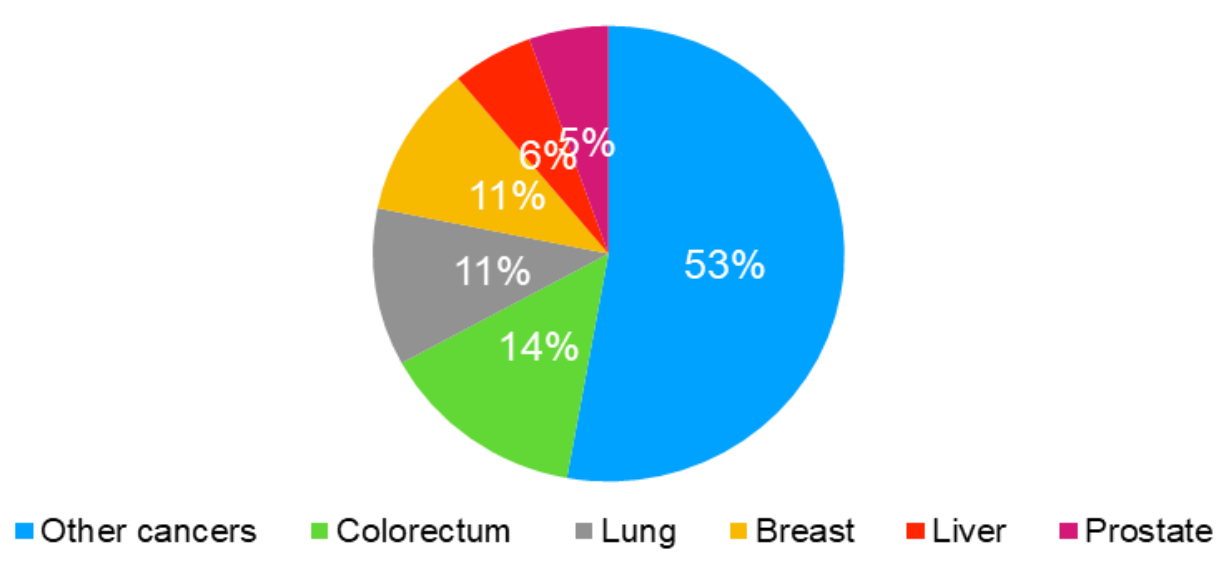

Figure 2 Number of new cases in the Republic of Moldova, 2018, both sexes

Source: International Agency for Research on Cancer 
In men in 2018, lung cancer with 1,323 new cases prevails, representing 16.6\% (in 2013 - 16.1\%), colorectal cancer with 1,241 new cases, representing 15.6\% (in 2013 - 13.0\%) and cancer prostate with 833 new cases, representing $10.4 \%$ (in $2013-9.6 \%$ ) (Figure 3 ).

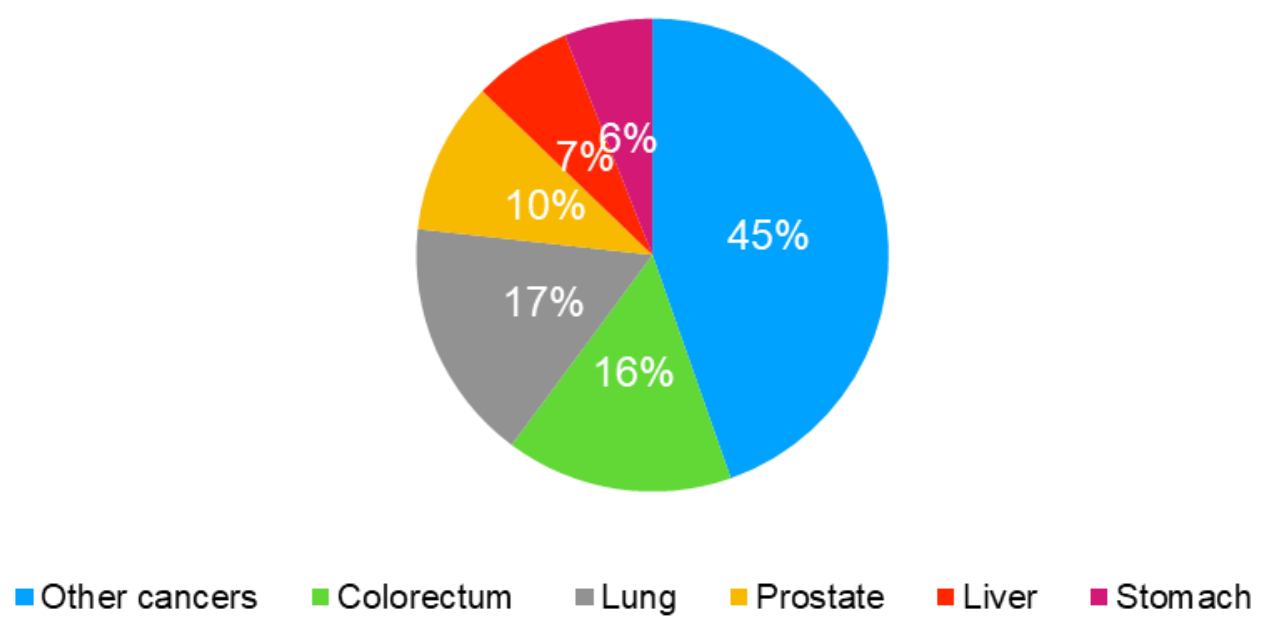

Figure 3 Number of new cases, 2018, men

Source: International Agency for Research on Cancer

In women, in the top of malignant tumor locations in 2018, breast cancer prevails with 1,646 new cases, representing $22.5 \%$ (in 2013 - 21.8\%), colorectal cancer with 930 new cases, representing 12.7\% (in 2013 - 11.3\%), cervical cancer with 639 new cases, representing 8.7\% (in 2013 - 7.3\%) (Figure 4).

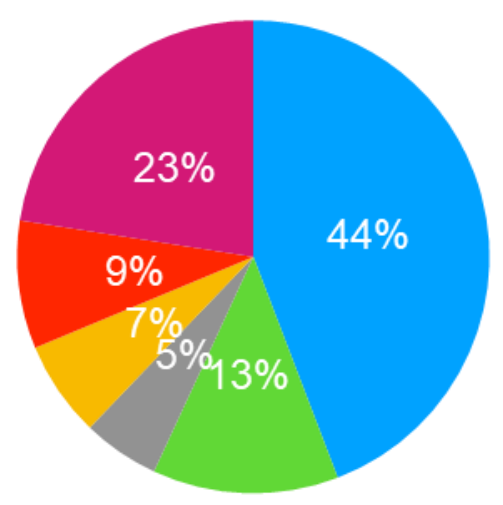

- Other cancers $\quad$ Colorectum $\quad$ Lung $\quad$ Corpus uteri $\quad$ Cervix uteri $\quad$ Breast

Figure 4 Number of new cases in the Republic of Moldova, 2018, women

Source: International Agency for Research on Cancer

The highest mortality rate for 2018 has lung cancer with 21.2 per 100,000 inhabitants, mammary gland cancer with 19.7 per 100,000 inhabitants and colorectal cancer with 18.7 per 100,000 inhabitants. (Figure 5). 


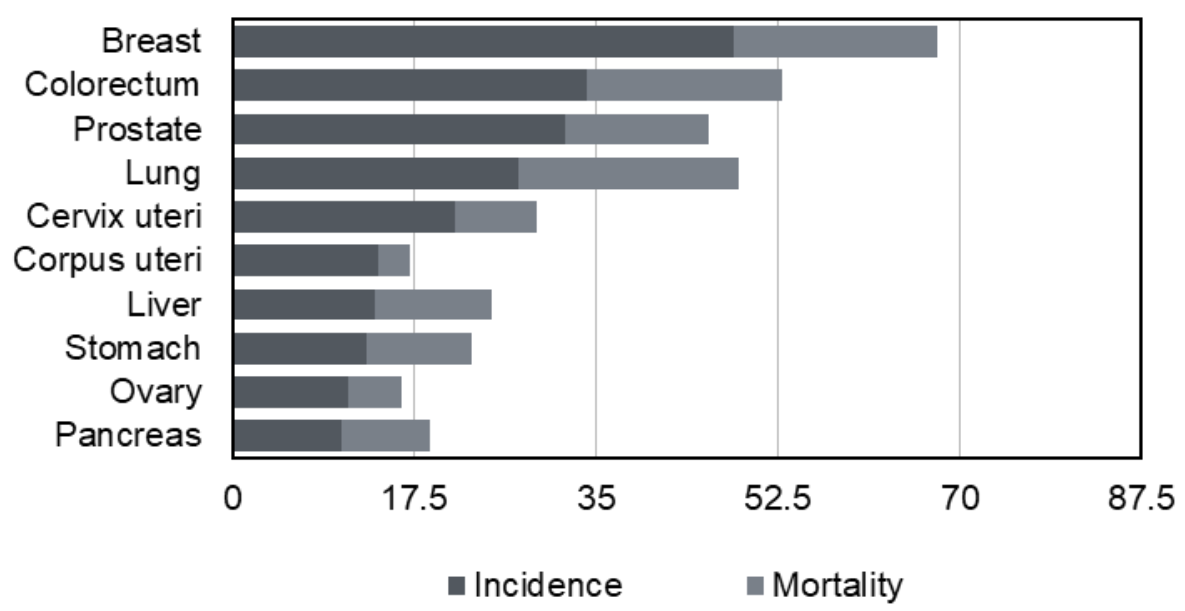

Figure 5 Incidence and mortality, top 10 cancers, 2018, Moldova

Source: International Agency for Research on Cancer

\subsection{Methods for evaluating the staging of patients with abdominal neoplasms}

The exact cancer stage is of fundamental importance for the selection and planning of treatment. Current staging paradigms focus primarily on a detailed delineation of the primary tumor in order to determine its resection capacity, and subsequently on assessing the presence of metastatic spread that would alter the surgical approach, or the mandate of non-surgical therapy. This approach is based on the assumption that the best, and sometimes the only, way to cure a cancer patient is surgical resection. Unfortunately, not all non-invasive techniques have the perfect ability to identify those primary tumors that are capable of being completely excised, nor the degree of their metastatic dissemination. However, due to relatively low costs and widespread availability, CT is the preferred scanning methodology for identifying tumors, local metastases, and spreading them remotely [10].

This technique is often complemented by other examinations that have improved their performance in staging areas. For example, magnetic resonance imaging (MRI), mammography, or ultrasound can be used as complementary T-stage examinations; surgical sampling of lymph nodes - for N-stage; bone scanning, MRI or ultrasound examination - for Mstage. Consequently, many patients undergoing a set of investigations are incorrectly organized based on the results obtained. Even for those who have resorted to surgery, only loco-regional metastases can be identified in resection specimens without the ability to detect distant metastases. As a result, many patients undergo unnecessary operations for pathologies that could never have been cured by surgery. In the case of restadialization, the situation is even more complicated. The sequelae of previous treatment can be difficult to differentiate from a residual cancer and the likelihood of patient rescue therapy is decreasing.

The definition of the malignant involvement degree is the foundation on which current oncological practice is based. This information defines applicable therapeutic strategies and provides a guide to the patient's prognosis. Diagnostic imaging methods, especially CT, are the primary techniques that are used to detect the cancer stage. These ways play a key role in cancer management.

Each of the different methodologies used to determine the cancer stage has inaccuracies. Due to its relatively low cost, widespread availability, and ability to define primary tumor relationships, lymph node drainage, and detect metastatic deposits in disparate tissues, CT scanning with contrast is administered the preferred methodology for the initial staging of the majority TNM [11]. Like other anatomical imaging techniques, diagnostic CT is generally good enough to demonstrate anatomical relationships of the primary tumor and, variably, whether it has traversed important tissue planes. However, normal anatomical distortion through local scarring, mechanical side effects of the primary tumor, such as obstructive atelectasis, or reactive changes in adjacent tissues, such as edema, can significantly compromise Tstage assessment. Moreover, lack of sufficient contrast between the tumor and many normal tissues makes it impossible for the CT to detect cases in which the primary tumor may cross a critical boundary that prevents it from being resected. Consequently, additional anatomical imaging studies such as MRI, mammography, and ultrasound may be used in combination with CT for staging of T. Even after such combinations of examinations, some patients cannot avoid operative evaluation to determine if resection is possible. 
As a result, additional diagnostic tests are often performed to assess certain sites of potential metastases in certain highrisk individuals. These tests may include liver ultrasound, brain MRI and bone scan. Thus, due to the limitations of diagnostic CT for TNM staging, many patients undergo a set of diagnostic examinations, each adding inconveniences, delays, costs and morbidity to the staging process. Even then, it was found that many patients were inadequately staged based on the results received.

From an imaging perspective, tumor progression is generally defined as an increase in tumor load (measured on imaging studies) or the detection of new lesions seen in imaging studies, and the tumor response refers to tumors that become smaller or disappear [12].

During the cytotoxic chemotherapy period, which began in the late 1940s-1950s, the "response rate" (RR), i.e. the proportion of tumors that decreased, was the main goal for clinical trials of anticancer therapies. Measurements of solid tumors were generally determined by imaging studies, and therefore guidelines, criteria, and classifications were given based on measurements from imaging studies developed in the 1980s and 1990s [13, 14]. These guidelines and classifications reflect the emphasis on the overall response rate, which was the norm at the time.

3D CT and MRI imaging has replaced standard radiography. Anatomical measurements can now be made much more accurately, and early metastatic lesions can be detected with much greater confidence in earlier stages than was the case even a decade ago.

Therefore, the measurements obtained from image scans must be as reproducible as possible. It should not matter what hospital a patient goes to or on what day, or on which manufacturer's scanner is scanned, the result should be the same. To achieve this, there must be a rigorous standardization and attention to detail on all hardware, software parameters, as well as the responsibility of human resources, which can introduce variability.

Many factors affect the target lesions measurement and the new lesions detection, ranging from the choice of imaging modalities, imaging acquisition techniques and image reconstruction parameters, to the variability related to the expertise and different measurement methods of physicians interpreting images [12].

Improper use of imaging, imaging technique and/or imaging reconstruction parameters in oncology clinical trials can lead to loss or delay in the detection of new (metastatic) lesions and thus misinterpretation of when a disease progresses.

Technical factors in the image acquisition process that are known to influence the measurement of the size of the lesion and therefore the evaluation of the anatomical response include differences in the technical parameters of the scanner, intravenous contrast, type of contrast, volume of contrast, timing, injection rate and CT scan beam settings [15 - 23]. During the scan acquisition the factors associated with the patient also play an important role, such as the respiration phase during which the image is acquired and whether or not the patient can suspend breathing. If the patient is unable to hold his breath for the entire scan ( $<30$ seconds), normal and abnormal structures may fade. This will generally cause the lesions to be measured larger than they really are and may result in complete loss of the small lesions.

Thus, in order to reduce the measurements variability, it is important that the CT scan images be reconstructed with the same slice thickness for a given patient.

Factors related to the radiologist interpreting the images are another significant source of variability in the interpretation and measurement of target lesions [24 - 29]. These factors include the expertise and ability of the radiologist in the quantitative and qualitative evaluation of the therapy response, measurement biases due to the systematic excess of the radiologist or under interpretation of tumor contraction, biases due to the radiologist's knowledge of treatment, discrepancies in measurements due to different patient descriptions by radiologists and human errors that can be caused by tracking different target lesions over time and overlooking the development of a new lesion. Furthermore, the measurement technique choice (e.g., one-dimensional, two-dimensional, volumetric), the measurement method used to determine tumor measurements (e.g., automated techniques), and the measurement environment (e.g., workstation display settings) may affect also the accuracy and reproducibility of a radiologist's measurements [30]. Difficult to measure lesions (for example, due to indistinct or obscure lesion edges, or lesions with heterogeneous densities) can further aggravate the lesion measurements accuracy and reproducibility .

Recent studies have begun to systematically assess the relative contribution of sources of variability to a better understanding of where corrective efforts should be directed to have the greatest impact. A prominent study is the same-day repeated CT examination, designed to investigate the minimal changes that could be detected by modern CT 
scanners using advanced measurement methods [19,22]. The study showed levels of measurement errors or variability in one-dimensional, two-dimensional and volumetric techniques. The smaller the lesion, the greater the measurement variability [22]. The results of these studies on sources of variability can not only help to determine the appropriate threshold values to distinguish real (biological) tumor changes from measurement errors (inherent variability of the technique), but also to identify and suggest the most appropriate acquisitions and reconstructions. imaging, parameters, and measurement techniques (e.g., one-dimensional, two-dimensional, or volumetric) to optimally monitor tumor response or progression.

In order to reduce the variability in the target lesions measurement and to improve the detectability of new lesions, continuous efforts are made for the continuous education of radiologists, standardization of image interpretation workflow, harmonization of imaging techniques and reconstruction parameters, improvement of visual capacity image, optimization of tumor measurement methods.

Methods for assessing tumor response to treatment have changed and continue to evolve [31 - 36]. The North American Radiology Society (RSNA) supports the Quantum Imaging Biomarkers Alliance (QIBA), which has published documents to standardize imaging acquisition for volumetric CT examination, FDG-PET, and contrast-enhanced dynamic MRI [37].

More consistent imaging strategies for tumor response include:

- Implement a scanner calibration program and evaluate the quality for each patient. Two such accreditation programs that can be used are the Centered Quantitative Imaging Excellence (CQIE) programs and the clinical trial network site qualification programs supported by SNMMI and scanner validation.

- The same radiologist evaluates the complete set of examinations for the same patient.

- The radiologist evaluates the images in the order in which they were obtained clinically (providing sets of images in an orderly manner over time).

- $\quad$ The same representative target lesions are measured at each scan [12].

The American Cancer Society (ACS) has recommended a screening of cancer patients every three years for people between the ages of 20 and 39, and annually for men and women between the ages of 40 and over [38]. However, as the routine controls intervals have been replaced by recommendations that apply to specific conditions and populations, the periodicity of a general health check when these case examinations could be performed has become less clear.

Contrast-enhanced CT or MRI examination plays a central role in long-term postoperative evaluation. The follow-up protocol includes imaging studies every 6 months for the first year and then at one-year intervals in negative cases. The monitoring interval is shorter (3 months) for intermediate lesions [39] and in patients undergoing chemotherapy [40].

Another source recommends evaluating the initial response at 4 weeks after surgery, with early detection of recurrence of recurrence by CT or MRI studies, every 3 months in the first 2 years and surveillance every 6 months later [41]. In case of tumor recurrence after curative treatments, re-evaluation of the patient should be performed using the staging system and treatment review [42].

\subsection{Post-processing programs in abdominal neoplasms}

As CT use has increased, concerns about the dose of the population by CT have begun to be expressed in the literature [43-46], making it clear that the responsible use of CT requires an adjustment of technical factors based on the size patient $[44,47,48]$. In response to these concerns, the radiology community (radiologists, medical physicists, equipment manufacturers) has implemented CT dose management procedures that correspond to the ALARA principle (As Low As Reasonably Achievable) $[45,49]$. The basic principle in selecting the right dose for a CT scan is that the attenuation of a particular patient and the specific diagnostic task must be considered. For large patients, the dose is higher than for small patients, which is in accordance with the ALARA principle.

In 1981, Haaga et al [50] published the concept of using the radiological tube current variation to reduce the radiation dose while maintaining image quality.

In 1994, GE Medical Systems put on sale the first radiological tube current modulation system, with which the dose was reduced by up to $20 \%$ [51]. In 1993 and 1994, Calendar et al [52,53] reported dose reductions of up to $40 \%$ in elliptical regions of the body, reductions achieved through the use of anatomical modulation of radiological tube current, which became available only at the end of 2001, when due to public concerns about the irradiation dose, its reduction has 
become a marketing tool. This development has forced the radiology community to define a variety of dose reduction products.

Another aspect would be that dose reduction strategies should be based not only on the use of dose optimization technology, but on the appropriate use of imaging [54]. A considerable part of CT scans could be replaced by alternative practical methods, or they could simply be eliminated. There is a possibility of reducing the use of many routine CT scans in favor of other diagnostic methods.

Here are some questions that every doctor should answer before recommending a radiological examination: What diagnostic procedure is most appropriate for a particular pathology in a particular patient? It is a clinically justified radiological procedure [55]. If so, which radiological procedure is most appropriate?

Optimizing the use of radiological examinations with the help of clinical decision guidelines is essential [55]. Once it has been established that an imaging procedure is clinically justified, the physician (and the entire imaging team) has the responsibility to optimize the patient's individual radiation exposure, which must be a continuous and regularly updated process.

Despite the extraordinary contributions of the CT scan to modern healthcare, special attention must be paid to the risk associated with ionizing radiation received during a CT scan.

In addition, each CT exam must be customized for each patient. Justification is a common responsibility between applicant clinicians and radiologists. Therefore, for medical exposures, the main tasks of the radiology community are to work with the team of clinicians to direct patients to the most appropriate imaging modality for the required diagnostic task, and to ensure that all technical aspects of the examination are optimized, so that the required level of image quality can be achieved while keeping the doses as low as possible. The American College of Radiology provides evidence-based guidance and appropriate criteria to assist physicians in recommending a necessary examination [56]. The European Commission and the Royal College of Great Britain presented a document with a detailed presentation of the clinical indications for imaging examinations, including the CT "Reference Guide for Radiologists" [57]. Thus, a CT scan should be performed only if the radiation dose is considered to be justified by the potential clinical benefit to the patient.

Since the cancer risk associated with radiation dose in CT is not zero is, it is clear that reducing the dose of radiation in CT examination must continue to be one of the main priorities of the radiological community, especially in light of the continuous increase in CT examinations performed annually [58].

The radiation dose in the CT scan can be quantified in a variety of ways. Output of radiation from the scanner, dose per organ and effective dose are the most common dose values [58].

The effective dose $[59,60]$, usually expressed in the unit of measurement $\mathrm{mSv}$, is a unit that represents a "whole body equivalent" of a dose that would have a similar health risk to that due to partial body irradiation. The actual dose allows for a rough comparison of risk-induced radiation between different types of examinations [62].

The actual dose is not a quantity that measures the radiation dose, but a concept that reflects the radiation risk stored in a particular patient. It should be emphasized that the current concept of effective dose is based on a mathematical model for a "standard" body [65], without age and sex specificity [66].

The irradiation dose is one of the most important determinants of the image quality of the CT examination and, therefore, the accuracy of the diagnosis and the result of a CT examination. The irradiation dose should be reduced only if the diagnostic image quality is not sacrificed. Therefore, in order to understand how the radiation dose in the CT scan can be reduced, it is necessary to become familiar with the relationship between image quality and radiation dose.

The dose reduction goal can be approached from the following two perspectives. The first perspective is to properly define the quality of the target image for each specific diagnostic task, which does not require low noise or higher spatial resolution than necessary.

The second perspective of dose reduction is to improve some aspects of image quality, such as image noise reduction, which can then be implemented in order to allow radiation dose reduction. This task can be accomplished by optimizing the techniques of the CT scanning system and improving the reconstructions processing . 
A huge effort has been made to improve the effective dose on CT systems, which is related to several components of this system, including detector, collimator and beam filter modeling.

A common method to optimize the radiation dose is to adapt the tube to the radiological current, using protocols based on weight or size [67 - 72]. A more advanced technique is Automatic Exposure Control (AEC), which aims at automatically modulating the current of the radiological tube to adjust for attenuation differences due to the patient's anatomy, shape, and size [73 - 77].

The intention of the AEC is to use the optimal level of radiation for each patient in order to obtain an adequate image quality related to a given diagnostic task. For smaller patients, less tube current, and therefore lower irradiation dose, is sufficient to achieve the desired image quality. For older patients, the radiation dose must be increased to ensure proper image quality.

Automatic exposure control systems are now available on major scanners. Although the basic principle of the ACS is the same, each is implemented somewhat differently in terms of the strategy for target image quality defining . CT users should be familiar with these techniques to ensure proper use. Improper use can lead to an increase in the patient's dose or a sacrifice in image quality.

\section{Conclusion}

The collective dose of the population resulting from medical imaging has increased sixfold in the last quarter of a century, so our goals should be to use imaging only when the potential clinical benefit outweighs the potential risk and strive for a imaging examination that provides the lowest dose required to obtain the desired information: we must aim at justifying and optimizing each imaging procedure.

The risks of exposure to iatrogenic radiation are often overlooked, and patients are rarely aware of these risks.

It is estimated that $30 \%$ of CT exams are unnecessary. The doctor requesting the examination must balance the risks and benefits, and the difficult cases should be discussed in a multidisciplinary clinical-radiological meeting for personalization of the radiological examination of cancer patients.

\section{Compliance with ethical standards}

\section{Acknowledgments}

I would like to express my deep gratitude to the scientific coordinator Prof. Rotaru Natalia and to the scientific consultant Prof. Caramella Davide, for the guidance and enthusiastic encouragement in the elaboration of this research.

\section{Disclosure of conflict of interest}

Nothing to declare.

\section{References}

[1] American Cancer Society. Global Cancer Facts \& Figures 4th Edition. Atlanta: American Cancer Society. 2018.

[2] National Institutes of Health (NIH). Understanding clinical studies [Internet]. October 18, 2016.

[3] Inskip PD, Curtis RE. New malignancies following childhood cancer in the United States, 1973-2002. International Journal of Cancer. 2007; 121: 2233-2240.

[4] Bhatia S, Yasui Y, Robison LL et al. High risk of subsequent neoplasms continues with extended follow-up of childhood Hodgkin's disease: report from the Late Effects Study Group. Journal of Clinical Oncology. 2003; 21: 4386-4394.

[5] Bhatti P, Veiga LH, Ronckers CX et al. Risk of second primary thyroid cancer after radiotherapy for a childhood cancer in a large cohort study: an update from the childhood cancer survivor study. Radiation Research. 2010; 174: 741-752.

[6] A Radiology Reporting Initiative [Internet]. 2016. 
[7] Practice guideline for communication of diagnostic imaging findings [Internet]. ACR. 2020.

[8] Ferlay J. Estimating the global cancer incidence and mortality in 2018: GLOBOCAN sources and methods. International Journal of Cancer. 2018.

[9] Béatrice Lauby-Secretan, Chiara Scoccianti, Dana Loomis et al. Body Fatness and Cancer - Viewpoint of the IARC Working Group. The New England Journal of Medicine. 2016; 375: 794-798.

[10] Rodney J Hicks, Robert E Ware, Eddie W F Lau PET/CT: will it change the way that we use CT in cancer imaging? Cancer Imaging. 2006; 6(Spec No A): S52-S62.

[11] Park Y, Colditz GA. Diabetes and adiposity: a heavy load for cancer. The Lancet Diabetes \& Endocrinology. 2018; 6: 82-83.

[12] Daniel Carl Sullivan, Lawrence H. Schwartz and Binsheng Zhao. The Imaging Viewpoint: How Imaging Affects Determination of Progression-Free Survival. Clinical Cancer Research. 2013; CCR-12-2936: 19(10).

[13] World Health Organization. WHO handbook of reporting result of cancer treatment. Geneva, Switzerland: JNCI: Journal of the National Cancer Institute. 2000; 92(3): 205-216.

[14] Therasse P, SG Arbuck, EA Eisenhauer, J Wanders. New guidelines to evaluate the response to treatment in solid tumors. European Organization for Research and Treatment of Cancer, National Cancer Institute of the United States, National Cancer Institute of Canada. Journal of the National Cancer Institute. 2000; 92: 205-16.

[15] Serup-Hansen E. Variation in gross tumor volume delineation using CT, MRI, and FDG-PET in planning radiotherapy of anal cancer. Journal of the Clinical Oncology. 30, 2012.

[16] Winer-Muram HT, S Gregory Jennings, Cristopher A Meyer. Effects of varying CT section width on volumetric measurement of lung tumors and application of compensatory equations. Radiology. 2003; 229: 184-94.

[17] Zhao B, Lawrence H Schwartz, Chaya S Moskowitz. Effect of CT slice thickness on measurements of pulmonary metastases - initial experience. Radiology. 2005; 234: 934-9.

[18] Petrou M, Leslie E Quint, Bin Nan, Laurence H Baker. Pulmonary nodule volumetric measurement variability as a function of CT slice thickness and nodule morphology. American Journal of Roentgenology. 2007; 188: 306-12.

[19] Zhao B, Leonard P James, Chaya S Moskowitz. Evaluating variability in tumor measurements from same-day repeat CT scans in patients with non-small cell lung cancer. Radiology. 2009; 252: 263-72.

[20] Reiner CS, Christoph Karlo, Henrik Petrowsky. Preoperative liver volumetry: how does the slice thickness influence the multidetector computed tomography- and magnetic resonance-liver volume measurements? Journal of Computer Assisted Tomography. 2009; 33: 390-7.

[21] Wang Y, ș.a. Volumetric measurement of pulmonary nodules at low-dose chest CT: effect of reconstruction setting on measurement variability. Eur Radiol. 2010; 20: 1180-7.

[22] Oxnard GR, Binsheng Zhao, Camelia S. Sima et al. Variability of lung tumor measurements on repeat computed tomography scans taken within 15 minutes. Journal of Clinical Oncology. 2011; 29: 3114-9.

[23] Tan Y, Pingzhen Guo, Helen Mann. Assessing the effect of computed tomographic (CT) slice thickness on unidimensional (1D), bidimensional (2D) and volumetric measurements of solid tumors. Cancer Imaging. 2012; 12: 497-505.

[24] Erasmus JJ, Gregory W Gladish, Lyle Broemeling. Interobserver and intraobserver variability in measurement of non-small-cell carcinoma lung lesions: implications for assessment of tumor response. Journal of Clinical Oncology. 2003; 21: 2574-82.

[25] Hopper KD, CJ Kasales, MA Van Slyke et al. Analysis of interobserver and intraobserver variability in CT tumor measurements. American Journal of Roentgenology. 1996; 167: 851-4.

[26] Wormanns D, Gerhard Kohl, Ernst Klotz et al.Volumetric measurements of pulmonary nodules at multi-row detector CT: in vivo reproducibility. European Radiology. 2004; 14: 86-92.

[27] Goodman LR, Meltem Gulsun, Lacey Washington. Inherent Variability of CT lung nodule measurements in vivo using semiautomated volumetric measurements. American Journal of Roentgenology. 2006; 186: 989-94.

[28] Punnen S, Massom A Haider, Gina Lockwood. Variability in size measurement of renal masses smaller than $4 \mathrm{~cm}$ on computerized tomography. Journal of Urology. 2006; 176: 2386-90. 
[29] Zhao B, Y Tan, DJ Bell. Exploring manual and computer-aided intra- and inter-reader variability in tumor unidimensional (1D), bi-dimensional (2D) and volumetric measurements. European Journal of Radiology. 11 Mar 2013.

[30] Schwartz LH, Ginsberg MS, DeCorato D et al. Evaluation of tumor measurements in oncology: use of film-based and electronic techniques. Journal of Clinical Oncology. 2000; 18: 2179-84.

[31] Eisenhauer EA, P Therasse, J Bogaerts et al. New response evaluation criteria in solid tumours: revised RECIST guideline (version 1.1). European Journal of Cancer. 2009; 45: 228-47.

[32] Byrne MJ, Nowak AK. Modified RECIST criteria for assessment of response in malignant pleural mesothelioma. Annals of Oncology. 2004; 15: 257-60.

[33] Ghassan K. Abou-Alfa, Lawrence Schwartz et al. Phase II study of sorafenib in patients with advanced hepatocellular carcinoma. Journal of Clinical Oncology. 2006; 24: 4293-300.

[34] Cheson BD, Beate Pfistner, Malik E. Juweid et al. Revised response criteria for malignant lymphoma. Journal of Clinical Oncology. 2007; 25: 579-86.

[35] Choi HA. Correlation of computed tomography and positron emission tomography in patients with metastatic gastrointestinal stomal tumors treated at a single institution with imatinib mesylate: proposal of new computed tomography response criteria. Journal of Clinical Oncology. 2007; 25: 1753-9.

[36] Lencioni R, LIovet JM. Modified RECIST (mRECIST) assessment for hepatocellular carcinoma. Seminars of Liver Disease. 2010; 30: 52-60.

[37] Buckler AJ, Linda Bresolin, N Reed Dunnick et al. A collaborative enterprise for multi-stakeholder participation in the advancement of quantitative imaging. Radiology. 2011; 258: 906-14.

[38] Dr. Robert A. Smith PhD, et al. American Cancer Society Guidelines for the Early Detection of Cancer. 2009.

[39] Arnold R, Yuan-Jia Chen, Frederico Costa et al. ENETS Consensus Guidelines for the Standards of Care in Neuroendocrine Tumors: follow-up and documentation. Neuroendocrinology. 2009; 90(2): 227-233.

[40] Oberg K, G Akerström, G Rindi. ESMO Guidelines Working Group. Neuroendocrine gastroenteropancreatic tumours: ESMO Clinical Practice Guidelines for diagnosis, treatment and follow-up. Annals of Oncology. 2010; 21(Suppl 5): v223-v227.

[41] Verslype C, Rosmorduc 0, Rougier P. Hepatocellular carcinoma: ESMO-ESDO Clinical Practice Guidelines for diagnosis, treatment and follow-up. Annals of Oncology. 2012 Oct; 23(suppl 7) vii 41-48.

[42] Anil Arora, Ashish Kumar. Treatment Response Evaluation and Follow-up in Hepatocellular Carcinoma. Journal of Clinical and Experimental Hepatology. 2014 Aug; 4(Suppl 3): S126-S129.

[43] BrennerD, C Elliston, E Hall, W Berdon. Estimated risks of radiation-induced fatal cancer from pediatric CT. American Journal of Roentgenology. 2001; 176: 289-296.

[44] Donnelly LF, K H Emery, A S Brody. Minimizing radiation dose for pediatric body applications of single-detector helical CT: strategies at a large children's hospital. American Journal of Roentgenology. 2001; 176: $303-306$.

[45] Haaga JR. Radiation dose management: weighing risk versus benefit. American Journal of Roentgenology. 2001; 177: 289-291.

[46] Nickoloff EL, Alderson PO. Radiation exposures to patients from CT: reality, public perception, and policy. American Journal of Roentgenology. 2001; 177: 285-287.

[47] Wilting JE, A Zwartkruis, M S van Leeuwen. A rational approach to dose reduction in CT: individualized scan protocols. European Radiology. 2001; 11: 2627-2632.

[48] Boone JM, Estella M. Geraghty, J. Anthony Seibert. Dose reduction in pediatric CT: a rational approach. Radiology. 2003; 228: 352-360.

[49] Linton OW, Mettler FA Jr. National conference on dose reduction in CT, with an emphasis on pediatric patients. American Journal of Roentgenology. 2003; 181: 321-329.

[50] HaagaJR, F Miraldi, W MacIntyre. The effect of mAs variation upon computed tomography image quality as evaluated by in vivo and in vitro studies. Radiology.1981; 138: 449-454. 
[51] Kalpana M. Kanal, Brent K. Stewart, Orpheus Kolokythas. Impact of Operator-Selected Image Noise Index and Reconstruction Slice Thickness on Patient Radiation Dose in 64-MDCT. American Journal of Roentgenology. 2007; 189: 219-225.

[52] Cynthia H. McCollough, Michael R. Bruesewitz, James M. Kofler, Jr. Dose reduction in CT by anatomically adapted tube current modulation: experimental results and first patient studies [abstract]. Radiology. 1997; 205 (P): 471.

[53] KalenderWA, Wolf H, Suess C. Dose reduction in CT by anatomically adapted tube current modulation: phantom measurements. Med Phys. 1999; 26: 2248-2253.

[54] Hedvig Hricak, David J. Brenner, S. James Adelstein. Managing Radiation Use in Medical Imaging: A Multifaceted Challenge. 2011.

[55] Radiation protection in medicine. ICRP publication 105. Annals ICRP. 2007; 37(6): 1-63.

[56] American College of Radiology. The American College of Radiology Appropriateness Criteria. 2008.

[57] Andre B. Bautista, Anthony Burgos, Barbara J. Nickel. Do Clinicians Use the American College of Radiology Appropriateness Criteria in the Management of Their Patients? American Journal of Roentgenology. 2009; 192: 1581-1585.

[58] Lifeng Yu, Xin Liu, Shuai Leng. Radiation dose reduction in computed tomography: techniques and future perspective. $2009 ; 1(1): 65-84$.

[59] Jacobi W. The concept of the effective dose - a proposal for the combination of organ doses. Radiat. Environ. Biophys. 1975; 12: 101-109.

[60] International Commission on Radiological Protection. Recommendations of the International Commission on Radiological Protection (ICRP Publication 26) Oxford, UK: The International Commission on Radiological Protection. 1977.

[61] McCollough CH, Schueler BA. Calculation of effective dose. Med. Phys. 2000; 27: 828-837.

[62] International Commission on Radiological Protection: 1990 Recommendations of the International Commission on Radiological Protection (Report 60) Annals of ICRP. 1991; 21: 1-3.

[63] AAPM Report No. 96: The Measurement, Reporting, and Management of Radiation Dose in CT. American Association of Physicists in Medicine (AAPM) Task Group. 2008; 23.

[64] National Council on Radiation Protection \& Measurements (NCRP) [Accessed 9 August 2009];Report no. 160: ionizing radiation exposure of the population of the United States (Press Release) 2009.

[65] Cristy M. Mathematical Phantoms Representing Children of Various Ages for Use in Estimates of Internal Dose. TN, USA: Oak Ridge National Laboratory.1980.

[66] DeMarco JJ, Cagnon CH, Cody DD, et al. Estimating radiation doses from multidetector CT using Monte Carlo simulations: effects of different size voxelized patient models on magnitudes of organ and effective dose. Phys. Med. Biol. 2007; 52: 2583-2597.

[67] Huda W, Bushong SC. In x-ray computed tomography, technique factors should be selected appropriate to patient size. Med. Phys. 2001; 28: 1543-1545.

[68] Frush DP. Strategies of dose reduction. Pediatr. Radiol. 2002; 32: 293-297.

[69] Huda W, Lieberman KA, Chang J, Roskopf ML. Patient size and x-ray technique factors in head computed tomography examinations. I. Radiation doses. Med. Phys. 2004; 31: 588-594.

[70] Huda W, Lieberman KA, Chang J, Roskopf ML. Patient size and x-ray technique factors in head computed tomography examinations. II. Image quality. Med. Phys. 2004; 31: 595-601.

[71] Nyman U, Ahl TL, Kristiansson M, Nilsson L, Wettemark S. Patient-circumference-adapted dose regulation in body computed tomography. A practical and flexible formula. Acta Radiol. 2005; 46: 396-406.

[72] McCollough CH. CT dose: how to measure, how to reduce. Health Phys. 2008; 95: 508-517.

[73] Gies M, Kalender WA, Wolf H, Suess C. Dose reduction in CT by anatomically adapted tube current modulation. I. Simulation studies. Med. Phys. 1999; 26: 2235-2247.

[74] Kalender WA, Wolf H, Suess C. Dose reduction in CT by anatomically adapted tube current modulation. II. Phantom measurements. Med. Phys. 1999; 26: 2248-2253. 
[75] Haaga JR, Miraldi F, MacIntyre W, et al. The effect of mAs variation upon computed tomography image quality as evaluated by in vivo and in vitro studies. Radiology. 1981; 138: 449-454.

[76] Lifeng Yu, Xin Liu, Shuai Leng et al. Radiation dose reduction in computed tomography: techniques and future perspective. Imaging Med. 2009 Oct; 1(1): 65-84.

[77] McCollough CH, Bruesewitz MR, Kofler JM., Jr CT dose reduction and dose management tools: overview of available options. Radiographics. 2006; 26: 503-512. 